\title{
Exploiting Spatiotemporal Information for Blind Atrial Activity Extraction in Atrial Arrhythmias
}

\author{
Francisco Castells ${ }^{1}$, Jorge Igual ${ }^{2}$, Vicente Zarzoso ${ }^{3}$, \\ José Joaquín Rieta ${ }^{1}$, and José Millet ${ }^{1}$ \\ ${ }^{1}$ Universidad Politécnica de Valencia \\ 46730 Gandia, Spain \\ \{fcastells,jjrieta,jmillet\}@eln.upv.es \\ ${ }^{2}$ Universidad Politécnica de Valencia \\ 03800 Alcoi, Spain \\ jigual@dcom.upv.es \\ ${ }^{3}$ Department of Electrical Engineering and Electronics, The University of Liverpool \\ Brownlow Hill, Liverpool L69 3GJ, UK \\ vicente@liv.ac.uk
}

\begin{abstract}
The analysis and characterization of atrial tachyarrhythmias requires the previous estimation of the atrial activity (AA) free from any ventricular activity and other artefacts. This contribution considers a blind source separation (BSS) model to separate the AA from multilead electrocardiograms (ECGs). Previously proposed BSS methods for AA extraction exploit only the spatial diversity introduced by the multiple electrodes. However, AA typically shows certain degree of temporal correlation, featuring a narrowband spectrum. Taking advantage of this observation, we put forward a novel two-step BSS-based technique which exploits both spatial and temporal information. The spatiotemporal BSS algorithm is validated on real ECGs from a significant number of patients, and proves consistently superior to a spatial-only ICA method. In real ECG recordings, performance can be measured by the main frequency peak and the spectral concentration. The spatiotemporal algorithm outperforms the ICA method, obtaining a spectral concentration of $58.8 \%$ and $44.7 \%$, respectively.
\end{abstract}

\section{Introduction}

Biomedical engineering is one of the research areas where the statistical tool of independent component analysis (ICA) has demonstrated a remarkable success. Indeed, ICA techniques are suitable to solve a large number of biomedical problems in electroencephalography (EEG) [1], magnetoencephalography (MEG), electrocardiography (ECG) [2], functional magnetic resonance imaging (fMRI) [3], etc. In the area of cardiac signal analysis, ICA methods can be employed for the separation of the ventricular activity (VA) and the atrial activity (AA). This separation is particularly useful in the study of atrial arrhythmias, e.g., atrial fibrillation (AF) or atrial flutter (AFL) [4], where AA and VA are temporally and spectrally overlapped.

The analysis and characterization of atrial arrhythmias from the ECG requires the previous estimation of AA. The main difficulty is that AA and VA appear mixed at the electrode outputs. The separation of these cardiac activities from the 12-lead standard ECG has already been modelled as a blind source separation (BSS) problem [5] although only spatial information has been utilized for imposing statistical independence in the estimated sources. Indeed, any prior information about the temporal structure of the sources is disregarded in most ICA algorithms applied to this problem. 
Motivated by the observation that VA and AA present specific spatiotemporal statistical properties, the present study presents a novel separation method more adapted to the biomedical problem in hand. In effect, VA can be modelled as a supergaussian distribution [6] whereas AA signals are typically quasi-gaussian and exhibit a narrowband spectrum with a main frequency of between $3.5-9 \mathrm{~Hz}$ [7][8]. Taking into account these considerations, a new BSS-based algorithm aiming to utilize more fully the spatiotemporal information of the ECG recordings is developed in a bid to enhance the quality of the estimated AA. The proposed approach combines ICA based on spatial-only higher-order statistics (HOS) with spatiotemporal second-order processing. The first stage is implemented with the FastICA method, whereas the second is carried out via the second order blind identification (SOBI) algorithm.

\section{Methods}

\subsection{Statistical Source Analysis}

The sources contained in an ECG recording can be divided into three types. VA sources are the ECG components with the highest energy. These components have a high amplitude during ventricular depolarization and repolarization (QRS complex and $\mathrm{T}$ wave respectively), but the rest of the time they present values close to zero due to the inactive period. Accordingly, VA sources possess supergaussian random distributions, even with kurtosis values above those of Laplacian distributions, as will be confirmed in the results section.

In the second place, AA consists of small and continuous wavelets with a cycle between $125 \mathrm{~ms}$ and $300 \mathrm{~ms}$. A statistical analysis of the sources shows that AA has kurtosis values very close to zero (as will be discussed later on), typical of quasigaussian distributions. AA waves have a characteristic spectrum, with a main peak due to the refractory period. This fact, which is neglected by practically all AA extraction methods to date, is exploited by the algorithm proposed in this paper. Fig. 1 shows an example of VA and AA waveforms, and their corresponding distribution estimates.

Finally, noise and other artefacts are the contributions with the lowest energy, although in more than a few leads they could show an amplitude of the same order of

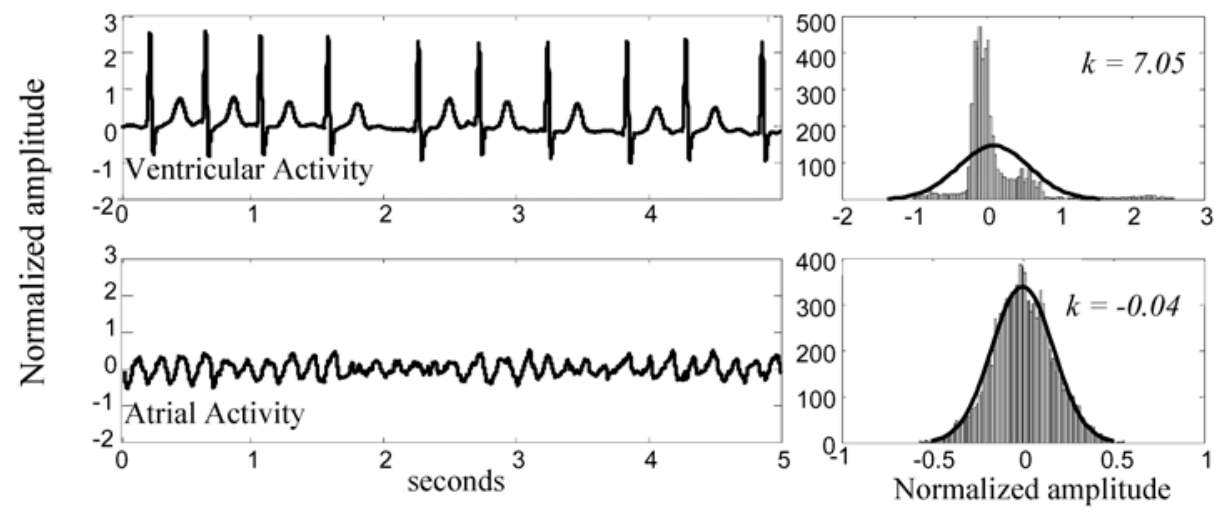

Fig. 1. Examples of VA and AA, and their histogram including kurtosis values. 
magnitude as the atrial sources, or even higher. The statistical behaviour of the noise may be different for each recording; even several noise sources with different statistical behaviour may be found in a single ECG. Hence, no assumption about the noise pdf or correlation can generally be made. The only noise assumption in the separation model we propose is that the noise and the AA source have different spectra. This hypothesis is verified in practically all cases.

\subsection{Two Stage Methodology}

The body-surface potentials as a result of cardiac electrical activity can be modelled as a blind source separation (BSS) problem:

$$
\mathbf{x}(t)=\mathbf{A s}(t)
$$

where $\mathbf{x}(t)$ is a length- $m$ vector which represents the electrode outputs at time instant $t$, $\mathbf{s}(t)$ is a length- $n(n \leq m)$ vector that represents the bioelectric sources, and $\mathbf{A}$ is the $m \times n$ mixing matrix which models the propagation from sources to electrodes. For the standard ECG, we have $m=12$. Neither the original sources nor the transfer coefficients from the epicardial surface towards the body surface are known.

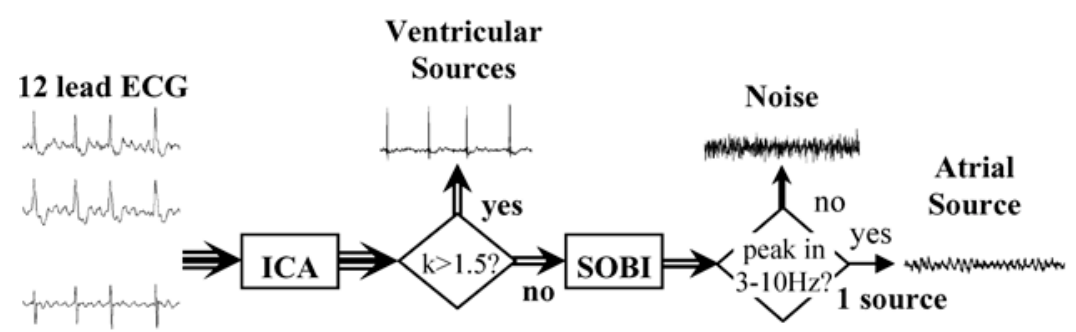

Fig. 2. Block diagram of the proposed hybrid approach to AA signal extraction.

In order to separate the AA free from VA and other interference, we propose a two-stage methodology, as illustrated in Fig. 2. The first stage exploits the supergaussian character of the VA to remove the ventricular contributions, and is implemented with spatial-only HOS-based ICA. Since ventricular components show the highest amplitude, this stage, if successful, eliminates the major source of interference. The remaining non-ventricular components (AA, artefacts and noise) are the inputs of the second stage. This step, which is implemented with spatiotemporal SOSbased ICA, takes advantage of the characteristic spectrum of AA in order to enhance the AA estimation quality.

\subsubsection{First Stage: Higher-Order Spatial Information (HOS-Based ICA)}

In general, ICA methods estimate a separation matrix $\mathbf{B}$ such that the estimated sources

$$
\hat{\mathbf{s}}(t)=\mathbf{B} \mathbf{x}(t)
$$

fulfil certain statistical independence criterion. HOS-based ICA techniques are most suitable to separate independent nongaussian sources. These techniques are able to 
estimate the independent sources by using certain measures of independence provided by the HOS of the multilead signal [9][10]. In this study we have chosen an algorithm that estimates nongaussianity as a function of the following approximation of negentropy $J(\cdot)[11]$ :

$$
\begin{aligned}
J(y) & \propto[\mathrm{E}[G(y)]-\mathrm{E}[G(v)]] \\
G(y) & =\log \cosh y
\end{aligned}
$$

where $y$ is the output signal and $v$ is a unit variance Gaussian variable. The maximisation of the contrast function can carried out, after pre-whitening, by means of a robust fixed point algorithm known as FastICA [12]. Note that the aim of this paper is not to emphasize the convenience of a specific ICA algorithm but to demonstrate the suitability of HOS-based ICA as a general concept for this first processing stage.

HOS-based ICA algorithms are especially equipped to extract all nongaussian sources, but are unable to separate gaussian sources since their HOS are null. Hence, all gaussian sources will appear mixed at the ICA output. The practical consequence over AF recordings is that VA sources will be correctly extracted, but the AA source can appear combined with gaussian-like sources of interference such as thermal noise and other artefacts. Due to the very low amplitude of the AA signal, the separation of AA from these sources of interference becomes an important necessary task. This task will be carried out in the second stage, which is described next.

\subsubsection{Second Stage: Second-Order Spatiotemporal Information (SOBI)}

The inputs to the second processing stage are the non-ventricular source components estimated by the first stage. The decision as to which components belong to the ventricular subspace and which components belong to the non-ventricular subspace can be done automatically. Due to the existence of the QRS complex, the ventricular sources are highly kurtic; by contrast, AA usually displays kurtosis values marginally different from zero. Consequently, a kurtosis-based threshold can be employed to distinguish between ventricular and non-ventricular sources. Preliminary experiments show that a conservative normalized-kurtosis threshold of around 1.5 allows us to retain the AA information in the non-ventricular subspace (the signal subspace which lies orthogonal to that spanned by the mixing-matrix columns associated to the ventricular sources) and reject all other sources that contain QRS complexes.

The so-called second-order blind identification (SOBI) is designed to separate a mixture of uncorrelated sources with different spectral content through a second-order statistical analysis which capitalizes on the source temporal information [13]. For this purpose, SOBI aims to find a transformation that simultaneously diagonalizes several correlation matrices at different lags. Since, in general, no transformation may exist that accomplishes such a stringent condition, a function that objectively measures the degree of joint approximate diagonalization (JD) at different lags is employed instead.

Let $\mathbf{z}$ denote the non-ventricular sources inaccurately estimated at the first processing stage, and $\mathbf{s}$ the associated actual sources, among which the desired AA source appears. In the simplified two-signal case, the real sources $\mathbf{S}$ and the whitened observations $\mathbf{z}$ are related through a Givens transformation:

$$
\mathbf{z}=\mathbf{Q s}, \quad \mathbf{Q}=\left[\begin{array}{cc}
\cos \theta & -\sin \theta \\
\sin \theta & \cos \theta
\end{array}\right]
$$


where $\theta$ is an unknown rotation angle. The rotation angle that maximizes the JD criterion allows the recovery of the original sources. The extension of this procedure to more than two signals is easily carried out through a Jacobi-like iteration. Full details are given in [13], and are omitted here due to the lack of space.

The SOBI algorithm is appropriate for extracting sources with a narrowband spectrum; hence its suitability for AA estimation. The number of matrices for joint diagonalization and their respective time lags must be properly selected. Since the autocorrelation of the AA source in AF episodes is quasi-periodic with a period around 160 ms - i.e., 160 samples at a sampling rate of $1 \mathrm{Khz}-$, correlation matrices with time lags comprising two cycles (that is, $320 \mathrm{~ms}$ ) are chosen. This choice guarantees that even for AF signals with larger AA cycle the lag range spans at least one complete cycle period. Choosing correlation matrices at evenly spaced lags of $20 \mathrm{~ms}$ (i.e., a total of 17 correlation matrices) guarantees a high proportion of significant (non-zero) autocorrelation values among the selected lags with an affordable computational complexity.

We refer to the proposed two-stage hybrid method as ICA-SOBI.

\section{Results}

28 ECGs digitised during $30 \mathrm{~s}$ at a constant sampling rate of $f_{s}=1 \mathrm{Khz}$ with 16-bit amplitude resolution were employed in our study. All patients were suffering from atrial arrhythmias, including $17 \mathrm{AF}$ and 11 AFL episodes. HOS-based ICA only (without the SOBI step) and ICA-SOBI were applied to this database. The estimation of the AA source was successful in all cases.

A spectral analysis was carried out in order to detect the main frequency $f_{\mathrm{p}}$. The AA source estimated with ICA provided the same main frequency as the AA source estimated with ICA-SOBI, being of $6.19 \pm 0.73 \mathrm{~Hz}$ for AF and $4.06 \pm 0.65 \mathrm{~Hz}$ for AFL. As an objective measure of AA extraction quality, the spectral concentration of the AA source around its main peak was computed according to the following expression:

$$
S C=\frac{\sum_{0.82 f_{p}}^{1.17 f_{p}} P_{\mathrm{AA}}\left(f_{i}\right)}{\sum_{0}^{f_{s} / 2} P_{\mathrm{AA}}\left(f_{i}\right)}
$$

where $P_{\mathrm{AA}}$ is the power spectrum of the AA signal, computed using the Welch's method over a 8192-point FFT with a 50\%-overlap 4096-sample Hamming window, and $f_{i}$ denote the FFT discrete frequency values.

The AA source obtained with ICA-SOBI had a higher spectral concentration around the main frequency peak in all cases. In average, ICA obtained a spectral concentration of $37.1 \%$ for AF and $54.5 \%$ for AFL. The spectral concentration was increased with ICA-SOBI up to $53.7 \%$ and $65.2 \%$ for AF and AFL, respectively. The higher spectral concentration of the AA signal obtained after SOBI processing indicates that part of the noise present in the AA signal after ICA is effectively removed by SOBI. Fig. 3 compares the spectral concentration levels of the estimated AA using both methodologies. A typical example of the estimated AA and its spectrum where ICA-SOBI outperforms ICA is shown in Fig. 4. 


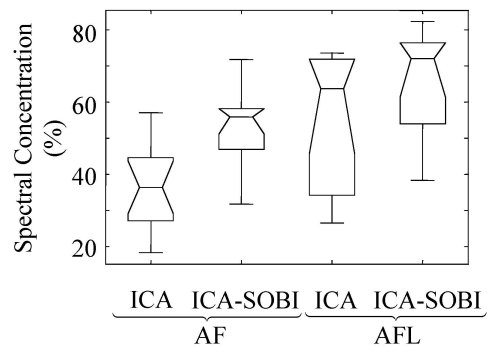

Fig. 3. Box-and-whiskers plot of the spectral concentration of the estimated AA.

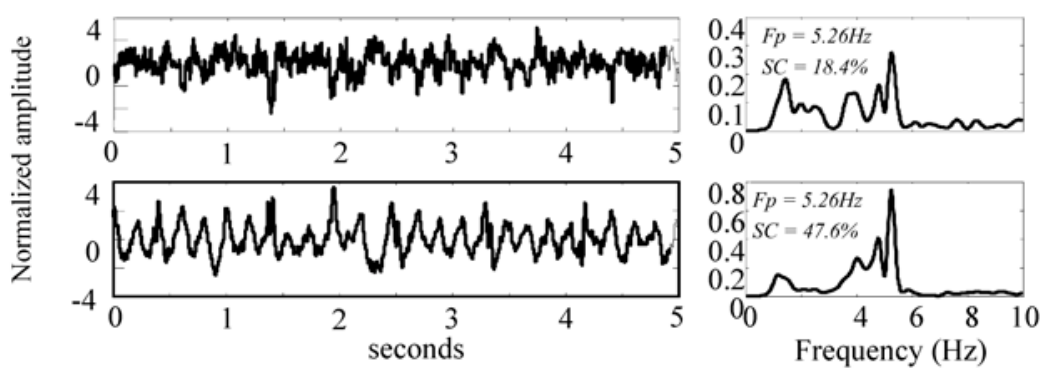

Fig. 4. An example where ICA-SOBI outperforms ICA.

Regarding the kurtosis values of the VA and the AA, the results confirm the hypotheses employed in the separation model. With a kurtosis value of $16.5 \pm 5.9$ for the ECGs under test, VA is indeed supergaussian. In contrast, AA cannot be assumed nongaussian, with a kurtosis value of $-0.21 \pm 0.45$ for this database. The fact that the estimated ventricular and atrial sources fulfilled the hypothesis assumed in the problem formulation regarding their statistical behaviour and spectral characteristics endorses the proposed approach for the enhanced estimation of AA in patients with atrial arrhythmias.

The improvement in the quality of the estimated AA after the latter stage appears closely correlated with the gaussianity of the sources. In the cases where the AA source presented a nongaussian character (i.e., kurtosis values significantly different from zero) the improvement in the spectral concentration was more important than in those cases with a higher gaussianity degree (kurtosis near zero). Fig. 5 illustrates the improvement in the spectral concentration as a function of the AA kurtosis.

\section{Discussion and Conclusions}

This paper has demonstrated that the source temporal information is indeed relevant in the estimation of AA from multi-lead ECG recordings of atrial arrhythmias episodes. A spatiotemporal BSS algorithm adapted to this specific problem has been designed and implemented. The algorithm consists of an initial spatial-HOS based separation stage (ICA) aiming to remove nongaussian interference (mainly VA), followed by a time-SOS based separation stage (SOBI) aiming to cancel gaussian-like noise. In this manner, the AA can be separated not only from VA, but also from other independent sources of noise and interference regardless of their distribution (gaus- 
sian or otherwise). An experimental study with real AF and AFL signals has validated the appropriateness of the proposed method.

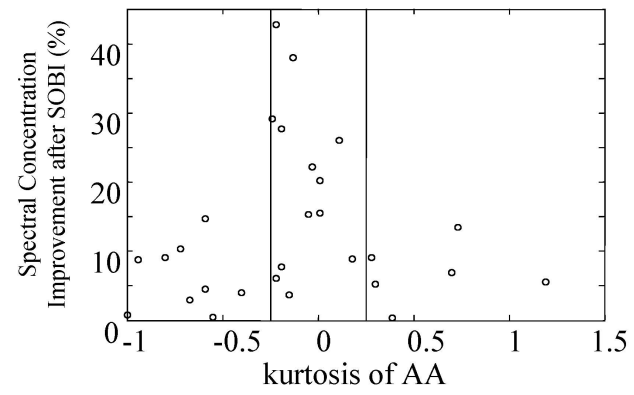

Fig. 5. Improvement in the spectral concentration as a function of AA source kurtosis.

In the experimental results, AA estimation has always improved with the application of the second separation stage exploiting temporal information. Even in ECGs where ICA had already estimated the AA accurately (because the existing AA was sufficiently nongaussian), the second step has been able to maintain the separation quality. Since the statistical behaviour of the AA source is not known a priori, but it may well change across patients, or even evolve in the same patient, it seems sensible to make use of the full two-step approach in all cases.

This contribution improves the existing solutions for AF analysis. Once the AA has been extracted, it can be further analyzed for spectral characterization, pattern recognition, time-frequency parameter extraction, etc. The proposed methodology thus emerges as a helpful tool in clinical diagnosis.

\section{Acknowledgements}

This study has been partly funded by the research grant TIC2002-00957 and the Universidad Politecnica de Valencia. V. Zarzoso is in receipt of a Post-doctoral Research Fellowship awarded by the Royal Academy of Engineering of the UK. This work was partially carried out while on leave at Laboratoire I3S, Sophia Antipolis, France. He gratefully acknowledges Pierre Comon's kind hospitality.

\section{References}

1. Makeig S., Bell A.J., Jung T.P., Sejnowski T.J., "Independent component analysis of electroencephalographic data", Advances in Neural Information Processing Systems, Vol. 8, 1996, pp. 145-151.

2. Barros A.K., Mansour A., Ohnishi N., "Adaptive blind elimination of artifacts in ECG signals", I\&ANN'98, Tenerife, Spain, Feb. 1998, pp. 1380-1386.

3. McKeown M.J., Makeig S., Brown G.G., Jung T.P., Kindermann S.S., Sejnowski T.J., "Analysis of fMRI data by blind separation into independent spatial components", Human Brain Mapping, Vol. 6, No 3, 1998, pp. 160-188.

4. Rieta J.J., Millet-Roig J., Zarzoso V., Castells F., Sánchez C., García-Civera G., Morell S., "Atrial fibrillation, atrial flutter and normal sinus rhythm discrimination by means of blind source separation and spectral parameters extraction", IEEE Computers in Cardiology, Memphis, Sep. 2002, pp. 25-28. 
5. Rieta J.J., Castells F., Sanchez C., Zarzoso V., Millet J., “Atrial activity extraction for atrial fibrillation analysis using blind source separation", IEEE Trans. Biomed. Eng., Vol. 51, 2004, pp. 1176-86.

6. Castells F., Igual J., Rieta J.J., Sánchez C., Millet J., "Atrial fibrillation analisis based on ICA including statistical and temporal source information", ICASSP-2003, Hong Kong, Apr. 2003, Vol. V, pp. 94-96.

7. Bollmann A., Kanuru N.K., McTeague K.K., Walter P.F., DeLurgio D.B., Langberg J.J., "Frequency analysis of human atrial fibrillation using the surface electrocardiogram and its response to ibutilide", Am. J. Cardiol. Vol. 81, 1998, pp. 1439-45.

8. Stridh M., Sörnmo L., Meurling C., Olsson B., "Characterization of atrial fibrillation using the surface ECG: Spectral analysis and timedependent properties", IEEE Trans. Biomed. Eng., Vol. 48, 2001, pp. 19-27.

9. Comon P., "Independent component analysis - a new concept?", Signal Processing, Vol. 36, 1994, pp. 287-314.

10. Cardoso J.-F., Souloumiac A., "Blind beamforming for non Gaussian signals", IEE Proceedings- $F$, Vol. 140, 1993, pp. 362-370.

11. Hyvärinen A., Karhunen J., Oja E., Independent Component Analysis, John Willey \& Sons, New York, 2001.

12. Hyvärinen A., "Fast and robust fixed-point algorithms for independent component analysis", IEEE Trans. on Neural Networks, Vol. 10, 1999, pp. 626-634.

13. Belouchrani A., Abed-Meraim K., Cardoso J.-F., Moulines E., "A blind source separation technique using second-order statistics", IEEE Trans. Sig. Proc., Vol. 45, 1997, pp. 434-444. 